\title{
Expression of lymphocyte immunoregulatory biomarkers in bone and soft-tissue sarcomas
}

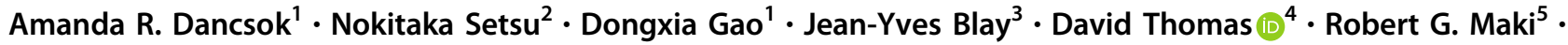 \\ Torsten O. Nielsen ${ }^{1}$. Elizabeth G. Demicco ${ }^{6}$
}

Received: 15 January 2019 / Revised: 29 May 2019 / Accepted: 30 May 2019 / Published online: 1 July 2019

(c) The Author(s), under exclusive licence to United States \& Canadian Academy of Pathology 2019

\begin{abstract}
Despite advances in our understanding of the underlying molecular drivers of sarcomas, few treatments are available with proven benefit for advanced metastatic sarcomas. Immunotherapy has value in this setting for some types of cancers, but sarcomas, with their multiplicity of rare types, have not been characterized in detail for their expression of targetable immune biomarkers. This study provides the most systematic evaluation to date of tumor-infiltrating lymphocytes and immune checkpoint biomarker expression in sarcomas. We examined by morphology and immunohistochemistry 1072 sarcoma specimens representing 22 types, in addition to 236 benign bone and soft-tissue tumors. Genomically-complex sarcoma types-those driven by mutations and/or copy-number alterations-had much higher numbers of tumor-infiltrating lymphocytes than translocation-associated sarcomas. Prior exposure to radiotherapy was associated with increased immune infiltrates. Higher lymphocytic infiltration was associated with better overall survival among the non-translocation-associated sarcomas. Expression of PD-1 and CD56 were associated with worse overall survival. LAG-3 and TIM-3, two emerging immune checkpoints, were frequently expressed in most sarcoma types. Indeed, most cases positive for PD-(L)1 coexpressed one or both of these novel biomarkers, providing a potential rationale in support for trials targeting LAG-3 and/or TIM-3 in conjunction with PD-1 inhibition.
\end{abstract}

Supplementary information The online version of this article (https:// doi.org/10.1038/s41379-019-0312-y) contains supplementary material, which is available to authorized users.

Torsten O. Nielsen

torsten@mail.ubc.ca

1 Department of Pathology and Laboratory Medicine, Vancouver Coastal Health Research Institute and University of British Columbia, Vancouver, BC, Canada

2 Department of Anatomic Pathology, Graduate School of Medical Science, Kyushu University, Fukuoka 812-8582, Japan

3 Department of Medical Oncology, Centre Léon Bérard and University Claude Bernard Lyon, Lyon, France

4 The Kinghorn Cancer Centre and Cancer Division, Garvan Institute of Medical Research, Darlinghurst, NSW, Australia

5 Northwell Health Monter Cancer Center and Cold Spring Harbor Laboratory, Lake Success, New York, NY, USA

6 Department of Pathology and Laboratory Medicine, Mount Sinai Hospital and Department of Laboratory Medicine and Pathobiology, University of Toronto, Toronto, ON, Canada

\section{Introduction}

Sarcomas are malignant tumors of the bone or soft tissue that demonstrate mesenchymal differentiation. The World Health Organization defines over 50 distinct sarcoma types that [1], while very heterogeneous in apparent histogenesis, tumor site, and molecular etiology, are most often treated primarily through surgery and radiation. Generally speaking, cytotoxic chemotherapy is not particularly effective for sarcomas, aside from some type-specific benefits [2, 3]. Despite advances in our understanding of the underlying molecular drivers of sarcomas and the development of rational therapies to target them, effective systemic treatment strategies for metastatic sarcomas, which occur in about half of patients [4], remain largely elusive. When metastasis occurs, cure is rare, resulting in a median overall survival (OS) of 12-18 months for advanced metastatic sarcomas [5-11]. New systemic treatment strategies are clearly needed, and immunotherapy provides a compelling approach.

Cancer immunotherapy is an emerging field encompassing both immunostimulatory and immunomodulatory 
approaches, which have shown remarkable promise in clinical trials, bringing about durable responses in some types of cancer previously considered "incurable." Immunostimulatory therapies, which function to initiate an antitumoral immune response, saw early successes in melanoma and leukemia [12-14], and have since shown efficacy in synovial sarcoma and myxoid/ round cell liposarcoma [15-18] in studies employing autologous $\mathrm{T}$ cells engineered with receptors to target cancer testis antigen NY-ESO-1. Immunomodulatory therapies, particularly those employing immune checkpoint inhibitors, have produced dramatic, practicechanging results in melanoma and lung and renal cell carcinomas [19-25]. Clinical trials of immune checkpoint inhibitors have yielded multiple successes in other tumor types, but few studies have been undertaken for sarcomas and no practice-changing benefits have been observed [26-31].

In the Phase 2 clinical trial of the anti-PD-1 checkpoint inhibitor pembrolizumab in sarcomas (SARC028) [26] and the Alliance randomized trial of nivolumab (anti-PD-1) \pm ipilimumab (anti-CTLA-4) [31], the most consistent and impressive responses were observed in patients with undifferentiated pleomorphic sarcoma, dedifferentiated liposarcoma, or myxofibrosarcoma. These findings generally support the widespread speculation that pleomorphic sarcomas, by nature of their higher mutational burden than translocation-associated sarcomas, exhibit increased immunogenicity, and are more likely to respond to immune checkpoint inhibitors than their genetically simpler counterparts. However, patients with some pleomorphic sarcomas, notably leiomyosarcoma or osteosarcoma, showed far less activity of PD-1-based immunotherapy. Though the relevance of PD-L1 expression to predict response to these agents remains unclear, studies in sarcomas generally report low, histotype-dependent expression of PD-L1 [32-35]. Recent publications have highlighted the existence of alternative immune checkpoint pathways [36-38], such as proapoptotic TIM-3 or antiproliferative LAG-3, that contribute to T-cell exhaustion and could explain resistance to checkpoint inhibitor monotherapy. Drugs targeting these pathways are in active development [38], and anti-TIM-3 antibodies have shown some promise in murine models of sarcoma in preclinical studies [39].

The purpose of this study is to characterize tumorinfiltrating lymphocytes across a large, comprehensive cohort of bone and soft-tissue sarcomas. We employ immunohistochemistry to identify important T-cell subsets within immune infiltrates and to assess expression of immune checkpoint biomarkers PD-1, PD-L1, TIM-3, and LAG-3. This study aims to describe, in a systematic manner, the expression of key immune checkpoint biomarkers across diverse sarcoma types.

\section{Materials and methods}

\section{Patient tumor samples}

Formalin-fixed, paraffin-embedded tissue microarrays were constructed at The University of British Columbia (Vancouver, BC, Canada) and at Mount Sinai Hospital (New York, NY, USA). From The University of British Columbia, 14 tissue microarrays were included: TMA-01-003 (synovial sarcoma and differential diagnoses, 82 cases in duplicate) [40]; TMA-03-008 (chondroid tumors, 121 cases in duplicate) [41]; TMA-06-001A (gastrointestinal stromal tumors, 148 cases in duplicate) [42]; TMA-06-007 (myxoid liposarcomas, 69 cases in triplicate) [43]; TMA-09-006 (epithelioid sarcoma and differential diagnoses, 53 cases in duplicate) [44]; TMA-10-004 (28 chordomas, in duplicate); TMA-10-009 (8 alveolar soft part sarcomas, 2 alveolar rhabdomyosarcomas, and 2 desmoplastic small round cell tumors, in triplicate) [44]; TMA-12-004 (BCL2-positive tumors, 35 cases in triplicate) [45]; TMA-12-005 (pediatric spindle cell lesions, 134 cases in duplicate) [45]; TMA-12006 (translocation-associated sarcomas, 10 cases in duplicate) [45]; TMA-12-010 (5 dedifferentiated liposarcomas and 5 undifferentiated pleomorphic sarcomas, in duplicate) [45]; TMA-14-006 (4 myxoid liposarcomas, 3 myxofibrosarcomas, 3 chondrosarcomas, 1 synovial sarcoma, and 1 malignant peripheral nerve sheath tumor, in duplicate) [46]; TMA-14-007 (dedifferentiated liposarcomas with welldifferentiated areas, both components for 57 cases in duplicate) [47]; and TMA-MPNST (malignant peripheral nerve sheath tumor and differential diagnoses, 176 cases in duplicate) [48]. From the Mount Sinai Hospital, three tissue microarrays were included: MSH-OSa (osteosarcomas, 280 cases in duplicate); MSH-SS (synovial sarcomas, 70 cases in duplicate); and MSH-UPS (75 undifferentiated pleomorphic sarcomas, 52 myxofibrosarcomas, 18 leiomyosarcomas, 13 dedifferentiated liposarcomas, 10 dermatofibrosarcoma protuberans, and differential diagnoses; 210 cases total in duplicate).

\section{Tissue microarray preparation}

All tissue specimens were derived from surgical resection specimens from Mount Sinai Hospital, New York, NY, USA ("MSH" tissue microarrays) [49], 20 centres throughout Norway (TMA-06-001), or Vancouver General Hospital, Vancouver, BC (all other tissue microarrays). Cores with a diameter of $1.0 \mathrm{~mm}$ (TMA-14-007, all Mount Sinai Hospital tissue microarrays) or $0.6 \mathrm{~mm}$ (all other tissue microarrays) were extracted from representative viable tumor tissue, as identified by a bone and soft tissue subspecialty pathologist (TON and EGD). Tissue microarrays were cut to $4-\mu \mathrm{m}$-thick sections, mounted to Fisherbrand ${ }^{\mathrm{TM}}$ Superfrost $^{\mathrm{TM}}$ Plus 
charged glass slides (Thermo Fisher Scientific Inc., Waltham, MA), and incubated for $1 \mathrm{~h}$ at $60^{\circ} \mathrm{C}$.

\section{Immunohistochemistry}

Immunohistochemical staining was performed on serial tissue microarray sections. All tissue microarray blocks were batch cut within 6 months of staining in order to prevent PDL1 degradation [50]. All Vancouver General Hospital tissue microarrays were stained within 7 days of batch cutting, and Mount Sinai Hospital tissue microarray blocks were cut immediately prior to shipping to UBC for staining. All antibodies, except for PD-L1, were applied using the Ventana DISCOVERY ${ }^{\circledast}$ ULTRA semiautomated staining system (Ventana Medical Systems Inc., Tucson, AZ), as described previously [47]. Briefly, heat-induced antigen retrieval was performed using the standard cell conditioning 1 (Ventana) protocol. Slides were incubated with primary antibodies (described in Table S1) in DISCOVERY antibody diluent (Ventana) for $2 \mathrm{~h}$ at room temperature. For CD4, CD8, CD56, and FOXP3, slides were incubated for $16 \mathrm{~min}$ at $37^{\circ} \mathrm{C}$ in DISCOVERY Universal secondary antibody (Ventana), and chromogen visualization was performed by DAB map detection (Ventana). For PD-1, LAG-3, and TIM3 , slides were incubated for $2 \mathrm{~h}$ at room temperature with the UltraMap anti-mouse (PD-1 and LAG-3) or anti-rabbit (TIM3) secondary antibody (Ventana) and visualized using the UltraMap DAB Kit (Ventana).

PD-L1 staining was performed using the Intellipath FLX automated staining system, as described previously [51]. Briefly, heat-induced antigen retrieval was performed using Diva Decloaker (Biocare Medical, Pacheco, CA, USA) for $30 \mathrm{~s}$ at $125^{\circ} \mathrm{C}$. Following the standard Intellipath FLX protocol, slides were blocked with Peroxidazed-1 (Biocare Medical) and Background Sniper (Biocare Medical), then incubated with primary antibody (Table S1) in DaVinci Green diluent (Biocare Medical) for $30 \mathrm{~min}$ at room temperature. Slides were incubated with secondary antibody (LLC MACH3 rabbit HRP-polymer, Biocare Medical) for $30 \mathrm{~min}$ at room temperature, followed by Intellipath FLC DAB chromogen kit (Biocare Medical) for $5 \mathrm{~min}$ at room temperature.

All slides were counterstained with hematoxylin and mounted. Digital images of immunostained tissue microarrays were acquired using the Olympus BLISS highdefinition virtual microscope and slide scanner (Olympus Life Science Solutions: Bacus Laboratories, Lombard, IL, USA) or the Aperio digital pathology slide scanner (Leica Biosystems, Wetzlar, Germany).

\section{Histological scoring}

Scoring was performed by pathologists experienced in scoring biomarkers in bone and soft-tissue tumors (NS, DG, and EGD). Replicate cores were scored separately, with the pathologist blinded to replicates and final histological diagnosis, and the mean score from all replicates was calculated. Tumor-infiltrating lymphocytes were counted directly from hematoxylin- and eosin-stained tissue microarray slides (Fig. S1A, B). Lymphocyte biomarkers (tumor-infiltrating lymphocyte counts, CD8, CD4, FOXP3, CD56, PD-1, TIM-3, and LAG-3) were scored by counting the number of positive-staining lymphocytes per tissue microarray core (Fig. S1C-J). Different core sizes were normalized by dividing scores by the area of the respective core, to give tumor-infiltrating lymphocytes per $\mathrm{mm}^{2}$. PD-L1 immunopositivity was scored in both the tumor fraction (by the percentage of positive sarcoma cells) and the lymphocyte fraction (by the count of positive lymphocytes). All immunohistochemical markers were scored for cytomembranous positivity, except for FOXP3 (a transcription factor), which was scored for nuclear positivity.

\section{Statistical analysis}

Data analysis was performed using IBM $^{\oplus}$ SPSS $^{\bullet}$ statistics software (version 26). An independent samples Kruskal-Wallis one-way ANOVA (analysis of variance) test was used to assess the differences in scoring between histological types. Categories were compared pairwise, and significance values were adjusted using the Bonferroni correction for multiple comparisons. Multivariable linear regression was used to assess the relative impact of biomarker scores and clinicopathological factors on survival. Survival correlates were evaluated using a Cox proportional-hazards multiple regression analysis to generate hazard ratios and corresponding $95 \%$ confidence intervals. Kaplan-Meier curves were generated based on cases positive for CD8 and/or FOXP3, and a log rank test was run to determine if there were differences in the survival distribution for the three predominant combinations or expression: CD8 - and FOXP3-, $\mathrm{CD} 8+$ and FOXP3 - , or CD8+ and FOXP3+. Statistically significant differences were defined as $p<0.05$.

\section{Results}

\section{Patient demographics}

In total, specimens from 1072 sarcomas (representing 22 histotypes, Table 1), 236 benign mesenchymal tumors, 33 carcinomas, and 21 melanomas were available for evaluation (Table S2). Of the 660 sarcomas for which detailed clinical and outcome data were available (Table 2), 209 (32\%) were classified as high grade (FNCLCC grade 3), 179 (27\%) as intermediate grade (FNCLCC grade 2), 61 (9\%) as low grade 
Table 1 Sarcoma samples on tissue microarrays

\begin{tabular}{|c|c|c|}
\hline Category & Sarcoma type & $N$ \\
\hline \multirow{9}{*}{$\begin{array}{l}\text { Translocation } \\
\text { associated }\end{array}$} & Synovial sarcoma & 177 \\
\hline & Myxoid liposarcoma & 39 \\
\hline & Ewing sarcoma & 21 \\
\hline & DFSP & 18 \\
\hline & Solitary fibrous tumor & 16 \\
\hline & Alveolar rhabdomyosarcoma & 10 \\
\hline & Alveolar soft part sarcoma & 8 \\
\hline & Low-grade fibromyxoid sarcoma & 8 \\
\hline & Clear cell sarcoma & 7 \\
\hline \multirow{14}{*}{$\begin{array}{l}\text { Mutation and/or } \\
\text { copy number driven }\end{array}$} & Osteosarcoma & 171 \\
\hline & GIST & 149 \\
\hline & MPNST & 76 \\
\hline & Chondrosarcoma & 71 \\
\hline & Well-differentiated liposarcoma & 67 \\
\hline & Dedifferentiated liposarcoma & 65 \\
\hline & UPS & 58 \\
\hline & Myxofibrosarcoma & 35 \\
\hline & Chordoma & 28 \\
\hline & Leiomyosarcoma & 21 \\
\hline & Embryonal rhabdomyosarcoma & 12 \\
\hline & Epithelioid sarcoma & 9 \\
\hline & Angiosarcoma & 4 \\
\hline & Total & 1072 \\
\hline
\end{tabular}

DFSP dermatofibrosarcoma protuberans, GIST gastrointestinal stromal tumor, MPNST malignant peripheral nerve sheath tumor, UPS undifferentiated pleomorphic sarcoma

(FNCLCC grade 1), and 215 had no grade specified (32\%). The median patient age was 45 (range 0-89), and the median tumor size was $7.0 \mathrm{~cm}$ (range $1-42 \mathrm{~cm}$ ). All of the 660 sarcomas with detailed outcome data were derived from surgical resection tissues. Prior to surgery, 380 patients (58\%) had not been treated by chemotherapy or radiotherapy, while 100 $(15 \%)$ had received neoadjuvant radiotherapy alone, $36(6 \%)$ had received neoadjuvant chemotherapy alone, and 16 (2\%) had received both. Therapy was not known in 128 cases (19\%). Certain sarcoma types were more likely to have been pretreated with neoadjuvant chemotherapy $(p<0.0005)$, e.g., osteosarcoma, and/or radiotherapy $(p<0.0005)$, e.g., dedifferentiated liposarcoma, Ewing sarcoma, alveolar and embryonal rhabdomyosarcoma, and synovial sarcoma, by Fisher's exact test.

\section{Characterization of tumor-infiltrating lymphocytes}

To obtain a broad picture of lymphocyte infiltration across our sample set, we first counted tumor-infiltrating
Table 2 Patient demographics. Clinical parameters and survival outcomes available for 665 cases. Median, quartiles (Q1-Q3), and range denoted for noncategorical variables

\begin{tabular}{|c|c|c|c|}
\hline & Median & Q1-Q3 & Range \\
\hline Age, years & 45.0 & $23-56$ & $1-89$ \\
\hline \multirow[t]{2}{*}{ Tumor size, $\mathrm{cm}$} & 7.0 & $4.0-7.5$ & $0.1-40$ \\
\hline & $N$ & $\%$ & \\
\hline \multicolumn{4}{|l|}{ Grade } \\
\hline 1 & 61 & 9.2 & \\
\hline 2 & 179 & 27.1 & \\
\hline 3 & 209 & 31.7 & \\
\hline Unknown & 211 & 32.0 & \\
\hline \multicolumn{4}{|l|}{ Neoadjuvant treatment } \\
\hline None & 380 & 57.6 & \\
\hline Radiotherapy & 100 & 15.2 & \\
\hline Chemotherapy & 36 & 5.5 & \\
\hline Chemo and radiation & 16 & 2.4 & \\
\hline Unknown & 128 & 19.4 & \\
\hline \multicolumn{4}{|l|}{ Adjuvant treatment } \\
\hline None & 296 & 44.8 & \\
\hline Radiotherapy & 80 & 12.1 & \\
\hline Chemotherapy & 59 & 8.9 & \\
\hline Chemo and radiation & 15 & 2.3 & \\
\hline Unknown & 210 & 31.8 & \\
\hline \multicolumn{4}{|l|}{ Local recurrence } \\
\hline Yes & 126 & 19.1 & \\
\hline No & 534 & 80.9 & \\
\hline \multicolumn{4}{|l|}{ Metastasis } \\
\hline Yes & 157 & 23.8 & \\
\hline No & 503 & 76.2 & \\
\hline
\end{tabular}

lymphocytes on haematoxylin and eosin-stained tissue cores. We observed wide differences in tumor-infiltrating lymphocyte counts based on sarcoma type, with dedifferentiated liposarcomas displaying distinctly higher levels of infiltrates than any other histological type (median 204 tumor-infiltrating lymphocytes $/ \mathrm{mm}^{2}$, Fig. 1a). We divided sarcoma types into two categories based on their characteristic genomic alterations: (1) translocationassociated sarcomas, and (2) mutation and/or copy number driven (nontranslocation) sarcomas (Table 1). The non-translocation-associated sarcomas as a group had significantly higher levels of tumor-infiltrating lymphocytes than translocation-associated sarcomas (median 54 lymphocytes $/ \mathrm{mm}^{2}$ vs. 34 lymphocytes $/ \mathrm{mm}^{2}, \quad p<0.0001$; Fig. 2a). Notably, chondrosarcoma exhibited the lowest lymphocyte counts overall (median 0 lymphocytes $/ \mathrm{mm}^{2}$; Fig. 1a), likely accounted for by the avascular nature of cartilage. The levels of tumor-infiltrating lymphocytes in translocation-associated sarcomas were more aligned with those observed in benign mesenchymal neoplasms, while 


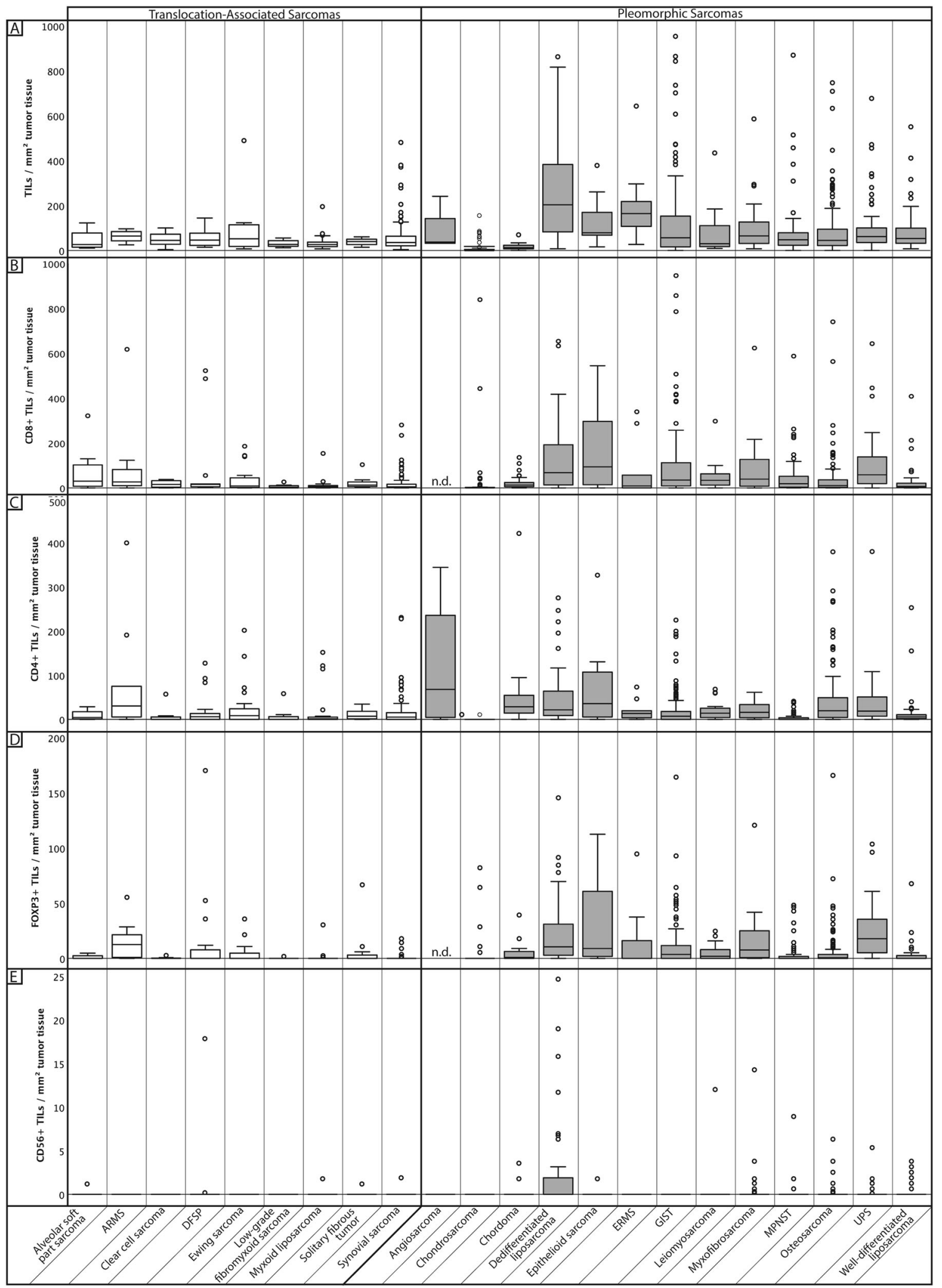

the profiles of the non-translocation-associated sarcomas corresponded more closely with those observed in carcinoma or melanoma (Fig. 2a).
We sought to subcategorize the infiltrating lymphocytes by immunohistochemistry, staining for cytotoxic $\mathrm{T}$ cells (CD8), helper T cells (CD4), regulatory T cells (FOXP3), 
Fig. 1 Sarcoma type-specific scores for: a tumor-infiltrating lymphocyte counts, b CD8, c CD4, d FOXP3, and e CD56. Boxplots depict comparative counts (per $\mathrm{mm}^{2}$ tumor tissue) of tumor-infiltrating lymphocytes from H\&E- or immunohistochemically-stained tissue microarrays. $y$-axis scales differ across figure. Abbreviations: ARMS, alveolar rhabdomyosarcoma; DFSP, dermatofibrosarcoma protuberans; ERMS, embryonal rhabdomyosarcoma; GIST, gastrointestinal stromal tumor; MPNST, malignant peripheral nerve sheath tumor; UPS, undifferentiated pleomorphic sarcoma; and TILs, tumor-infiltrating lymphocytes

and natural killer cells (CD56). Counts for all lymphocyte subsets were again highest among the non-translocationassociated sarcomas (Fig. 2b), particularly dedifferentiated liposarcoma (Fig. 1b-e). CD8+ lymphocytes were the most numerous lymphocyte subset across most sarcoma types, whereas CD56+ lymphocytes were scarce, with a median score of $0 / \mathrm{mm}^{2}$ across all types (Figs. $1 \mathrm{~b}-\mathrm{e}$ and $2 \mathrm{~b}$ ). We used serial section staining to examine coinfiltration by multiple lymphocyte subtypes, defining a positive case for each marker as one having at least one positive-staining lymphocyte in any tissue microarray core. Across the non-translocation-associated sarcomas, $50 \%$ of cases demonstrated the presence of CD8+ lymphocytes, CD4+ lymphocytes, and FOXP3+ lymphocytes (but not CD56+ lymphocytes, Fig. 2c), while $17 \%$ were positive for $\mathrm{CD} 8$

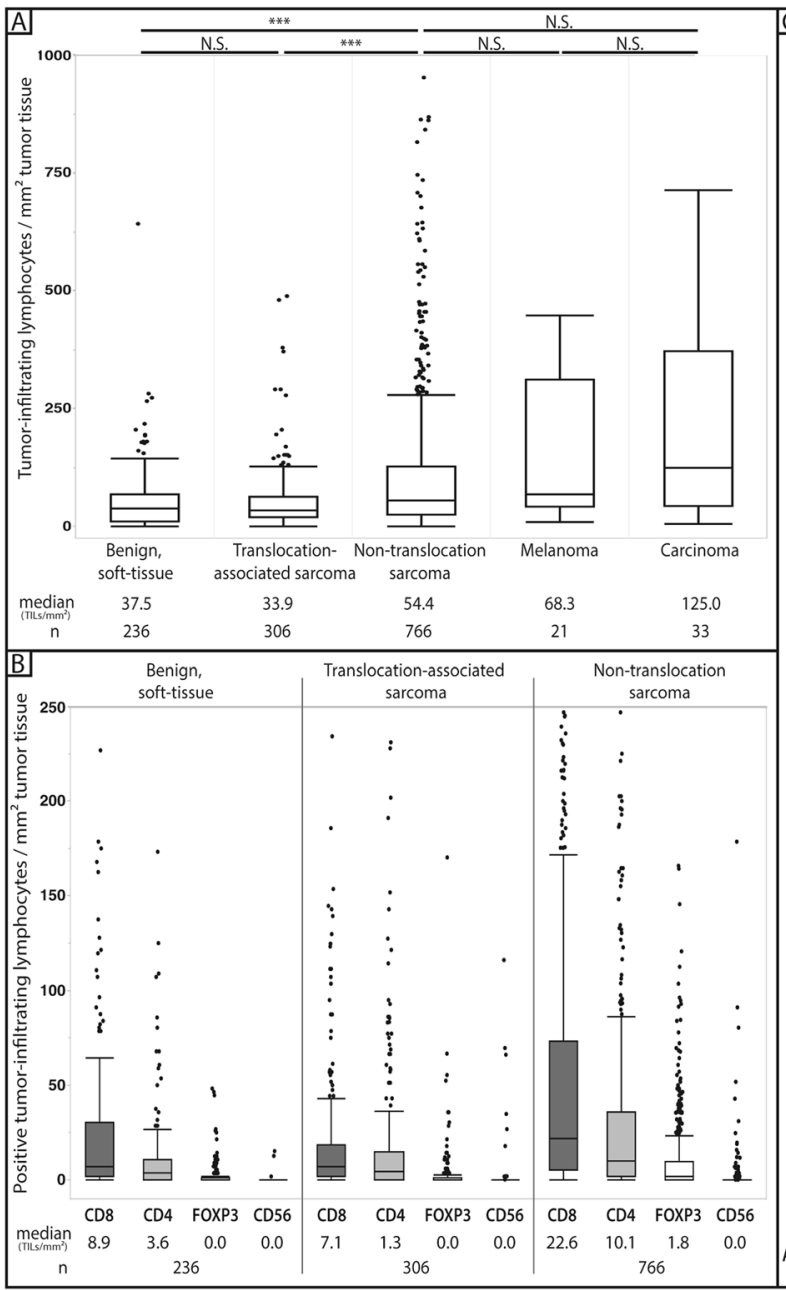

Fig. 2 a Boxplots depicting comparative counts of tumor-infiltrating lymphocytes from H\&E-stained tissue microarray cores of benign softtissue neoplasms, translocation-associated sarcomas, and mutation and/or copy number driven (nontranslocation) sarcomas, melanomas, and carcinomas. Boxes represent the first through third quartiles, horizontal line indicates median, and whiskers indicate range. Extreme outliers are indicated as dots. b Boxplots depicting counts of tumorinfiltrating lymphocytes staining positive for CD8 (cytotoxic T cells), CD4 (helper T cells), FOXP3 (natural killer cells), or CD56 (natural killer cells) in tissue microarray cores of benign soft-tissue neoplasms, translocation-associated sarcomas, or non-translocation-associated

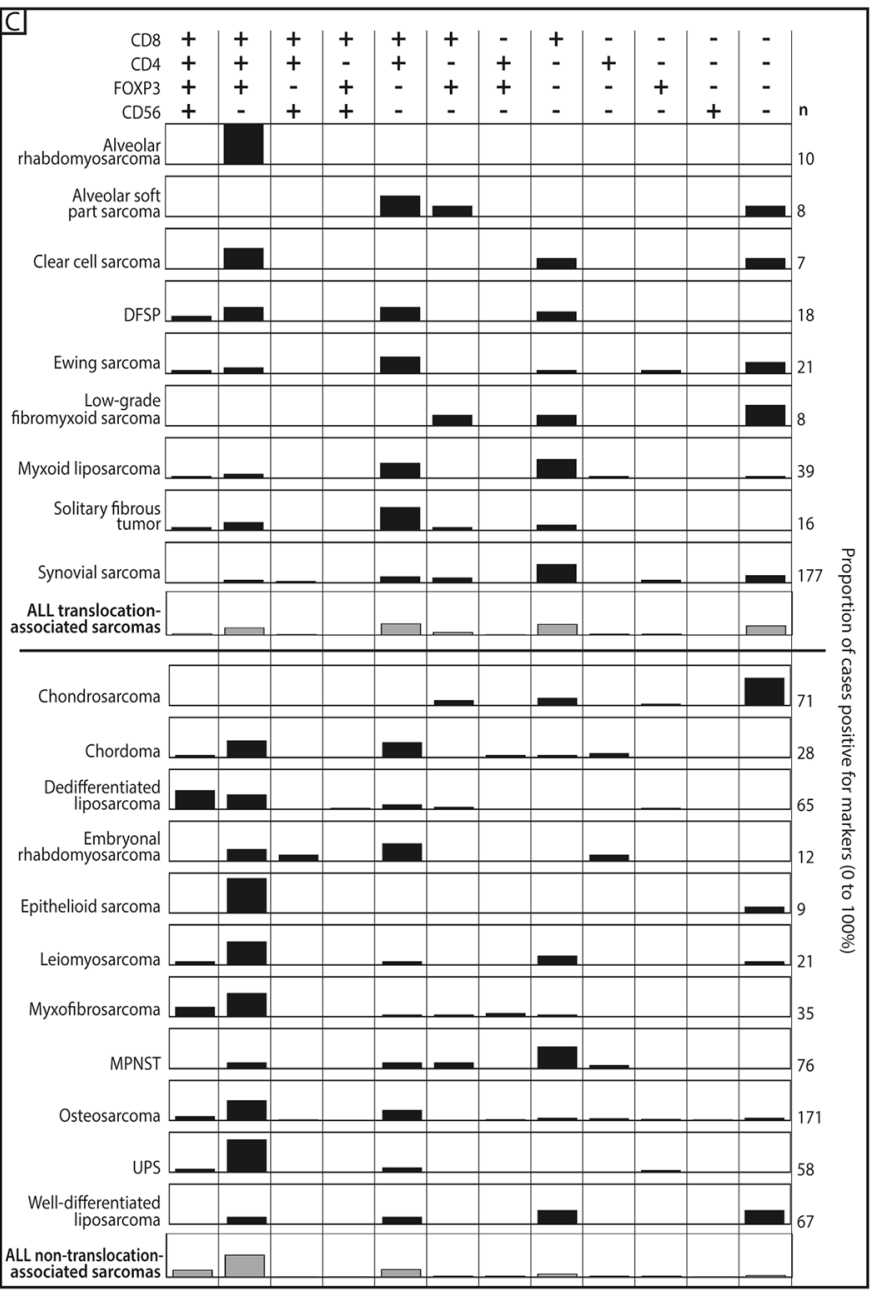

sarcomas. c Proportion of cases positive for one or more of CD8, CD4, FOXP3, and CD56 (by immunohistochemistry on tissue microarray samples, positive is defined as at least one positive-staining lymphocyte in any tissue microarray core). Panels represent histological type or translocation/non-translocation-associated classification. Angiosarcoma is not included here because scores for these four markers were not all available for any of the $(n=4)$ cases due to core dropout. Asterisks indicate $p<0.0001$, nonsignificant differences are denoted by "N.S.". Abbreviations: DFSP, dermatofibrosarcoma protuberans; MPNST, malignant peripheral nerve sheath tumor; and UPS, undifferentiated pleomorphic sarcoma 
only and $15 \%$ were positive for all four lymphocyte markers. Cases staining positive for all four markers were mostly osteosarcomas or myxofibrosarcomas. Comparatively, translocation-associated sarcomas were more likely to have no lymphocytic infiltrates (21\% of cases) or to be infiltrated by CD8 + lymphocytes, with ( $25 \%$ of cases) or without (24\% of cases) CD4+ lymphocytes (Fig. 2c).

To clarify the impact of tumor grade and of prior exposure to radiation or chemotherapy on the immune infiltration of sarcomas, we ran a multivariate regression analysis for the 660 cases for which clinical data were available. Taking into account grade and neoadjuvant treatment, the distinction of non-translocation- vs. translocation-associated sarcoma was independently predictive of increased tumorinfiltrating lymphocytes per $\mathrm{mm}^{2}$ of tissue $(p=0.002)$, as well as increased CD8 $+(p=0.03)$ and CD4 $+(p<0.001)$ tumor-infiltrating lymphocytes (Table S3). Tumor grade did not correlate with lymphocyte infiltration or expression of any tumor-infiltrating lymphocyte subset, except for FOXP3, which showed a positive association (Table S3). Prior exposure to radiation was independently predictive of increased $\mathrm{CD} 8+, \mathrm{CD} 4+$, and FOXP3+ tumor-infiltrating lymphocytes $(p=0.006, p<0.001$, and $p<0.001$, respectively; Table S3), but neoadjuvant chemotherapy was only associated with increased FOXP3 + tumor-infiltrating lymphocytes ( $p=0.001$; Table S3). CD56 showed no associations with any clinical parameters (Table S3).

\section{Expression of immune checkpoint biomarkers}

We next investigated expression of the targets of immune checkpoint inhibitors: PD-1, PD-L1, LAG-3, and TIM-3. Expression of PD-1 and PD-L1, targets of established checkpoint inhibitor drugs, was infrequent, being observed in only 10 and 22\% of sarcoma cases, respectively. Among all sarcomas, PD-1-positive lymphocyte counts ranged from $0 / \mathrm{mm}^{2}$ to $168 / \mathrm{mm}^{2}$, and PD-L1 positive tumor cell percentages from 0 to $95 \%$, with most sarcoma types showing a median of 0 PD-1-positive lymphocytes and 0 PD-L1 positive tumor cells (Fig. S2A, B). The least frequent expression of PD-1 and PD-L1 was seen in gastrointestinal stromal tumors (2\% of cases positive) (Figs. 3a and S2A, B). Emerging targets of immune checkpoint inhibitor therapy, LAG-3 and TIM-3, were expressed in 42 and 54\% of sarcoma cases, respectively (Figs. 3a and S2C, D). Positive cases, considered as the presence of at least one positive-staining lymphocyte on any tissue microarray core, were most common among the non-translocationassociated sarcomas, particularly dedifferentiated liposarcoma (77\% LAG-3+, 88\% TIM-3+), myxofibrosarcoma (68\% LAG-3+, 85\% TIM-3+), undifferentiated pleomorphic sarcoma (73\% LAG-3+, 85\% TIM-3+), and leiomyosarcoma (58\% LAG-3+, 74\% TIM-3+) (Figs. 3 and S2).
Coexpression of at least two immune checkpoints occurred in $29 \%$ of sarcomas overall, with $11 \%$ of sarcomas positive for all three checkpoint biomarkers, and $16 \%$ positive for LAG-3 and TIM-3 but not PD-1 (Fig. 3b). Of cases with PD$1+$ infiltrates, $77 \%$ also had LAG-3+ tumor-infiltrating lymphocytes and $84 \%$ had TIM-3+ tumor-infiltrating lymphocytes. The large majority of translocation-associated sarcomas $(64 \%)$ were negative for all three markers, with $9 \%$ positive for LAG-3 only, $12 \%$ positive for TIM-3 only, and $6 \%$ positive for both (but not PD-1). Non-translocationassociated sarcomas demonstrated triple positivity in $16 \%$ of cases, with $20 \%$ positive for both LAG-3 and TIM-3, 9\% positive for LAG-3 only, and $20 \%$ positive for TIM-3 only (Fig. 3b). A total of $29 \%$ of non-translocation-associated sarcomas were negative for all three markers.

By multivariate regression analysis, the distinction of nontranslocation- vs. translocation-associated sarcoma was only associated with increased expression of PD-1 $(p=0.02)$, but not of the other immune checkpoint biomarkers (Table S3). Grade and neoadjuvant therapy did not show a correlation with immune checkpoint biomarker expression (Table S3).

\section{Prognostic implications}

We ran a multivariate Cox proportional hazards model including age, grade, histological diagnosis, and scores for tumor-infiltrating lymphocyte and checkpoint markers. Among the non-translocation-associated sarcomas, overall and progression-free survival were marginally better with increasing lymphocytic infiltration $(p=0.02$ and $p=0.01$; Fig. 4a). OS was worse with increasing numbers of CD56+ tumor-infiltrating lymphocytes $(p=0.03)$ and PD-1+ tumor-infiltrating lymphocytes ( $p=0.05$; Fig. 4a). Among the translocation-associated sarcomas, tumor-infiltrating lymphocyte counts and immune checkpoint biomarker expression did not show any significant associations with overall or progression-free survival.

We generated Kaplan-Meier curves, classified by CD8 and FOXP3 expression, into three groups: (1) no tumorinfiltrating lymphocytes, (2) CD8+ lymphocytes only, and (3) CD8+ and FOXP3+ lymphocytes. The pleomorphic sarcoma subtypes as a group, defined here as the combined histotypes of angiosarcoma, dedifferentiated liposarcoma, leiomyosarcoma, myxofibrosarcoma, and undifferentiated pleomorphic sarcoma, showed improved OS with increased lymphocytic infiltrates (hazard ratio $=$ $4.50, p=0.034$; Fig. 4c). In dedifferentiated liposarcoma -for which no cases were negative for tumor-infiltrating lymphocytes-patients positive for both CD8 and FOXP3 had better OS than those negative for FOXP3 (hazard ratio $=9.46, p=0.002$; Fig. $4 \mathrm{~d}$ ), a pattern also observed in malignant peripheral nerve sheath tumor (hazard ratio $=12.66, p=0.002$; Fig. 4e). The inverse pattern was 


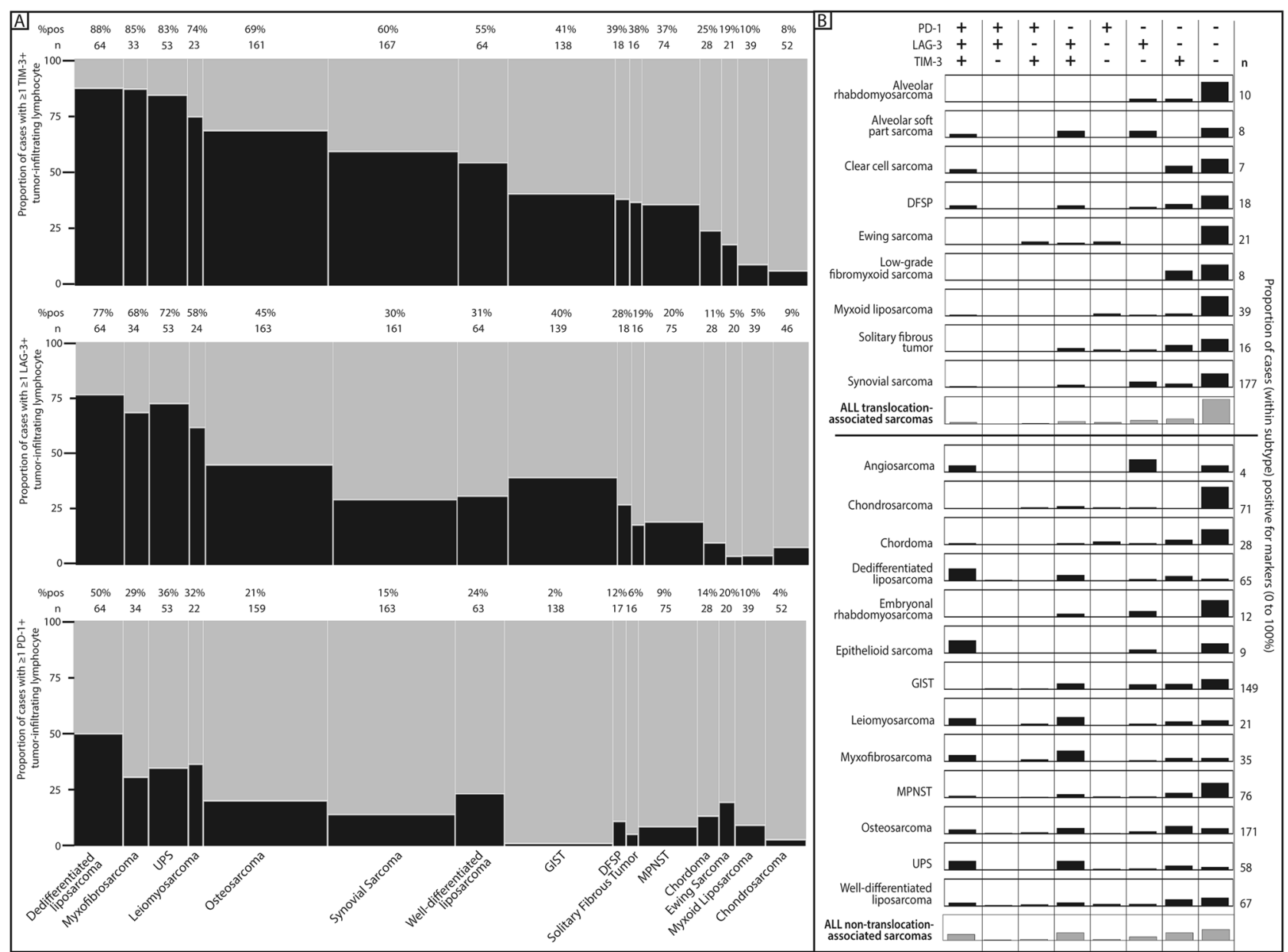

Fig. 3 a Mosaic plots comparing the proportion of cases positive for TIM-3, LAG-3, and PD-1 (by immunohistochemistry) across sarcoma types. Black bars indicate cases that are positive for each marker. A case is marked positive if there is at least one positive-staining lymphocyte in any tissue microarray core. Column width is proportional to the number of cases assessed, height to the fraction of positive cases. Abbreviations: UPS, undifferentiated pleomorphic sarcoma; GIST, gastrointestinal stromal tumor; DFSP, dermatofibrosarcoma protuberans; and MPNST, malignant peripheral nerve sheath tumor. b Proportion of cases positive for one or more of immune checkpoint

observed in myxoid liposarcoma, wherein cases positive for both CD8 and FOXP3 had worse OS than those with only CD8 positivity (hazard ratio $=32.00, p<0.001$; Fig. 4f). Translocation-associated sarcomas as a group did not demonstrate any significant associations. The same trends were observed for progression-free survival as OS, but statistical evaluations did not reach significance.

\section{Discussion}

The effectiveness of systemic treatment for many types of sarcoma remains unsatisfactory [52, 53], and implementation of immunotherapy trials for sarcomas lags behind biomarkers PD-1, LAG-3, and TIM-3 (by immunohistochemistry on tissue microarray samples, positive is defined as at least one positivestaining lymphocyte in any tissue microarray core). Panels represent histological type or translocation/non-translocation-associated classification. Positive is defined as at least one positive-staining lymphocyte per $\mathrm{mm}^{2}$ of tumor tissue on tissue microarray. Abbreviations: DFSP, dermatofibrosarcoma protuberans; MPNST, malignant peripheral nerve sheath tumor; and UPS, undifferentiated pleomorphic sarcoma

other, more prevalent diseases. This study provides a systematic characterization of tumor-infiltrating lymphocytes and immune checkpoint biomarker expression across the broadest range of sarcoma types examined to date, bridging the, thus, far uneven coverage-in terms of both histotypes and biomarkers assessed-by studies examining the sarcoma immune microenvironment [32, 33, 35, 54-67].

Our analysis indicates that non-translocation-associated sarcomas (mutation and/or copy-number driven) are more likely to demonstrate T-cell immune infiltrates than translocation-associated sarcomas. In particular, dedifferentiated liposarcoma, as well as myxofibrosarcoma, undifferentiated pleomorphic sarcoma, and epithelioid sarcoma, had the most numerous and diverse populations of tumor- 


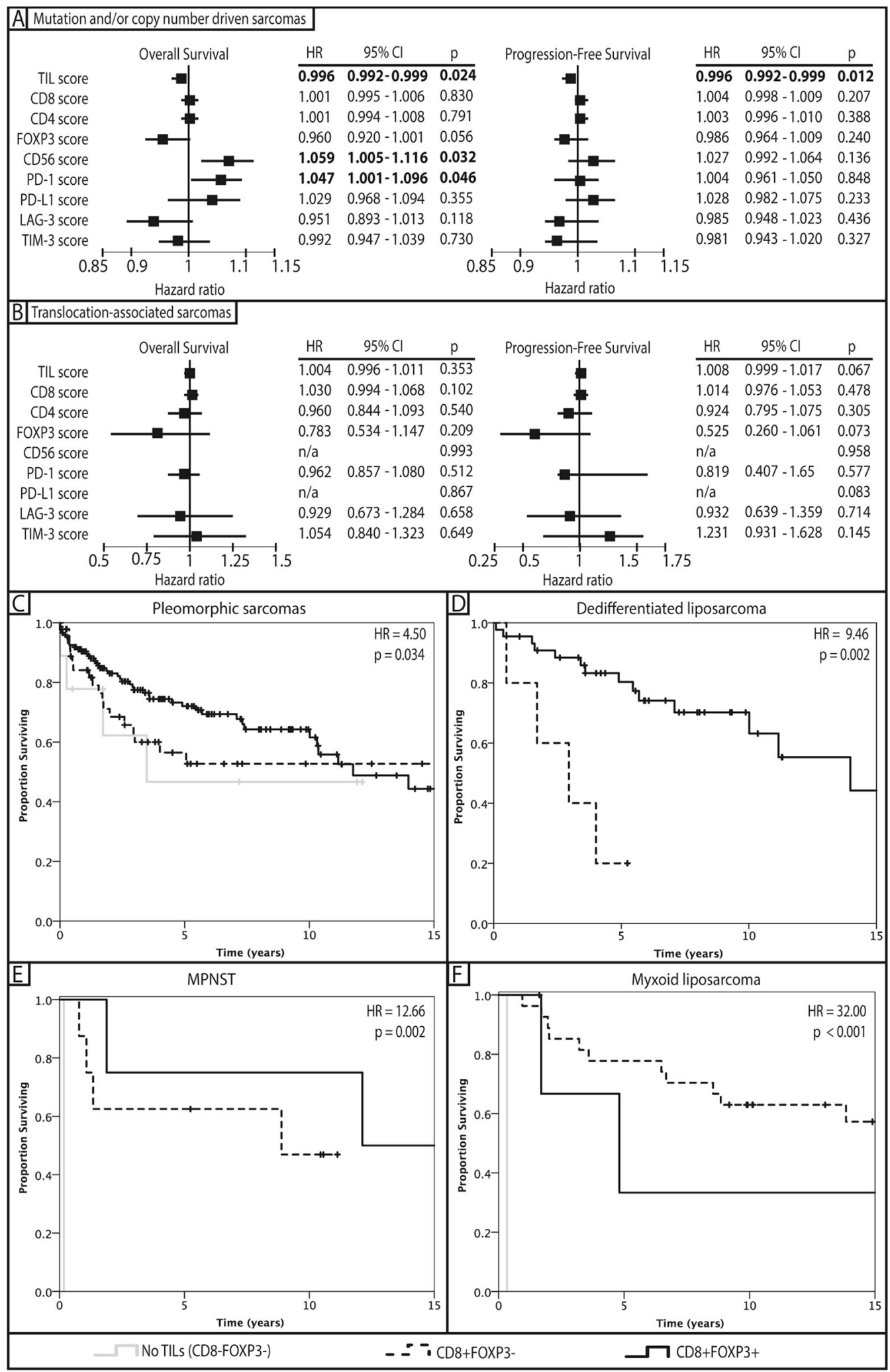

infiltrating lymphocytes among the 23 investigated sarcoma types. Our observation that non-translocation-associated sarcomas present an immune microenvironment more like that of melanoma or carcinoma-index indications for immune checkpoint blockade-suggests that these sarcomas are the most suitable candidates for such therapies. This 
Fig. 4 Forest plots depicting hazard ratios (and 95\% confidence intervals) of tumor-infiltrating lymphocytes and lymphocyte subset staining for overall survival across a mutation and/or copy-number driven (nontranslocation) sarcomas or $\mathbf{b}$ translocation-associated sarcomas. Hazard ratios were determined by Cox proportional-hazards multiple regression analysis. Age, grade, and sarcoma histotype were included in multiple regression analyses, but are not shown in plots; Kaplan-Meyer curve for overall survival in c pleomorphic sarcomas (angiosarcoma, dedifferentiated liposarcoma, leiomyosarcoma, osteosarcoma, and undifferentiated pleomorphic sarcoma), d dedifferentiated liposarcoma, e malignant peripheral nerve sheath tumor, or f myxoid liposarcoma. Curves are stratified according to presence of tumor-infiltrating lymphocyte subsets by immunohistochemical positivity for: CD8 (cytotoxic T cells), and/or FOXP3 (natural killer cells) in tissue microarray cores. Abbreviations: HR, hazard ratio; 95\% CI, 95\% confidence interval; and TIL, tumor-infiltrating lymphocyte

finding agrees with what has been observed in early clinical trials of immune checkpoint blockade in sarcomas [26, 2830, 68].

The driving stimulus for the numerous T-cell infiltrates in non-translocation-associated sarcomas remains unclear. In pan-cancer analyses, overall leukocyte fraction correlated positively with mutational burden, neoantigen counts, and intratumoral heterogeneity across a wide spectrum of malignancy, but negatively with measures of copy number alteration [69]. However, non-translocation-associated sarcomas are largely copy-number driven tumors, with low mutational loads and predicted neoantigens relative to most carcinomas [69]. In fact, within the subset of nontranslocation-associated sarcomas analyzed by The Cancer Genome Atlas (TCGA) (leiomyosarcoma, undifferentiated pleomorphic sarcoma, myxofibrosarcoma, and malignant peripheral nerve sheath tumor) [70], copy number alteration measured as fraction of bases deviating from baseline ploidy correlated negatively with leukocyte fraction (Spearman Rho $-0.4193, p<0.0001$ ), while no significant correlation with leukocyte fraction or CD8 $+\mathrm{T}$ cells was identified for other measures of DNA damage (e.g., intratumoral heterogeneity, aneuploidy score, mutational burden, or predicted neoantigens) [69]. Thus, it is likely that other molecular events trigger the influx of lymphocytes in this setting, possibly including dysregulation of cytokines resulting in increased inflammatory infiltrates, or alterations in specific genes contributing to immunogenicity [69].

Unlike the other non-translocation-associated sarcomas, osteosarcoma and leiomyosarcoma have had disappointing results in clinical trials of checkpoint inhibitors [26, 27, 30, 31, 71]. Our data suggests that these sarcomas are associated with sparse lymphocytic infiltrates, potentially explaining why immunomodulatory therapy has been largely unsuccessful. Our data are largely congruent with findings reported in TCGA study, where leiomyosarcomas showed lower median mRNA expression of CD8 relative to undifferentiated pleomorphic sarcoma/myxofibrosarcoma and dedifferentiated liposarcoma [70]. Further, data from the TCGA pan-cancer analysis indicates that leiomyosarcomas have a lower overall leukocyte fraction, relative to other pleomorphic sarcomas. As expected, translocationassociated sarcomas displayed the lowest overall levels of tumor-infiltrating lymphocytes across our dataset, mirroring the immune microenvironment of benign lesions rather than that of the non-translocation-associated sarcomas. This implies that translocation-associated sarcomas are immunoquiescent, despite high expression of immunogenic cancer testis antigens in many of these sarcomas, e.g., synovial sarcoma and myxoid liposarcoma [15-18], and therefore immunostimulatory therapy might be a more appropriate avenue for these sarcomas than would be checkpoint inhibition. Consistent with this idea, translocation-associated sarcomas have had some success with cancer vaccine [72, 73] and adoptive T-cell strategies [17, 18, 74]. Conceivably, some of the non-translocation-associated sarcoma types with low immunogenicity, such as chordoma, might also do well on similar regimens. Of note, several clinical trials of immune checkpoint inhibitors in carcinoma have reported that tumor mutational burden might be a biomarker of response to PD-L1/CTLA-4 blockade [75-77]. Sarcomas, in contrast, typically have low mutational burden, yet some have responded to immune checkpoint inhibitors, suggesting that different clinical biomarker predictors of response might be needed in this context.

In subtyping tumor-infiltrating lymphocytes, we observed a large predominance of CD8+ (cytotoxic) $\mathrm{T}$ cells. This suggests that the immune system is aware of the tumor and poised to activate antitumor immunity, but is being rendered inert by one or more immunoregulatory mechanisms. The presence of effector lymphocytes bodes well for the potential success of immune checkpoint blockade in these tumors. Conversely, the near absence of CD56+ tumor-infiltrating lymphocytes suggests that natural killer cell-mediated immune therapies might not be particularly suitable for sarcomas.

PD-1-positive lymphocytes were observed in $13 \%$ of translocation-associated sarcoma, and $20 \%$ of nontranslocation-associated sarcomas, and PD-L1-positive tumor cells in 3 and $12 \%$, respectively, findings contrasting with some studies [33-35, 78-81], but agreeing with others [32, 82-87]. The variable expression of these markers between our study and others may reflect the decay of PD-L1 in older tissue blocks [50, 88], as many of the tumor cases used to create our tissue microarrays were $>5$ years old, as well as the lower sensitivity of the anti-PD-L1 antibody (SP142) used in this study compared to other clones, such as 22C3 [89, 90]. Moreover, both tumor cell expression of PD-L1 and lymphocyte expression of PD-1 throughout the tumor may be heterogeneous, which can also account for potential discrepancies in scoring between the 
tissue microarrays used in different studies. Of note, one of the limitations of immune checkpoint studies in sarcoma is that sarcomas tend to be large, often exceeding $10 \mathrm{~cm}$ in size, and may be heterogeneous. Thus not only tissue microarray cores but also whole section staining may underscore PD-L1 expression in large tumors if negative. The implications of this in determining therapeutic eligibility for PD-1 inhibitors is as yet uncertain.

LAG-3 and TIM-3 are emerging immune checkpoints that have yet to be investigated in sarcomas [38]. Expression of both of these biomarkers was frequently seen across all sarcoma types, and, in our sample set, most sarcomas expressing PD-1 also expressed one or both of LAG-3 and TIM-3. The biologic significance of this finding is uncertain; while LAG-3 has been suggested to synergize with PD-1 to mediate T-cell exhaustion and tumor immune escape in some contexts [91], this has not been shown in sarcoma. We hypothesize that similar to other diseases where a compensatory upregulation of TIM-3 and LAG-3 following treatment with immune checkpoint monotherapy may occur [92-94], sarcomas may also benefit from combined inhibition of LAG-3 or TIM-3 together with PD-1. Future trials in sarcoma immunotherapy should aim to pursue these alternative immune checkpoints, alone or preferably in combination with other immune checkpoint inhibitors.

In addition to the associations identified between immune infiltration and sarcoma type, we observed that neoadjuvant radiation therapy has an independently significant association with a higher quantity of tumorinfiltrating lymphocyte subsets. This might be a result of radiation-induced immunogenic cell death, wherein dying cells release damage-associated molecular patterns that attract immune infiltrates [95, 96]. This phenomenon has been recently discussed as a potential key mechanism of the abscopal effect, the phenomenon by which localized radiation triggers shrinkage of distal tumor sites and metastases [97]. Our data suggests that the abscopal effect may well be relevant in sarcomas, highlighting the potential to observe a benefit in clinical trials that combine immune checkpoint inhibition with radiotherapy.

Likely due to the small numbers of cases with demonstrable immune infiltration, OS among the translocationassociated sarcomas did not associate with any of the immune markers in this study. Within our non-translocation-associated sarcoma samples, increasing tumor-infiltrating lymphocyte counts associated with improved overall and progression-free survival, suggesting that the presence of an immune response in these histologies has a survival benefit. Higher counts of CD56+ tumor-infiltrating lymphocytes, thought to represent the natural killer subset of lymphocytes, were associated with worse OS among the non-translocation-associated sarcomas, which seemingly contradicts the expected role of natural killer lymphocytes in targeting cancer cells. However, CD56+ tumor-infiltrating lymphocytes were rare across our entire sample set, so it might be that the few tumors that did attract natural killer cells were a subset of particularly aggressive sarcomas. PD-1 expression also associated with worse OS in this group, presenting an immunoevasive environment that is likely contributing to worse outcomes.

New systemic therapy options are needed for sarcomas, and immuno-oncology approaches have engendered a great deal of excitement, with many drugs becoming approved for a variety of neoplastic indications. This study contributes to the body of knowledge that might help better select the sarcomas patients most likely to benefit from particular immunotherapy approaches, such as those targeting LAG-3 or TIM-3. Deepening our understanding of the diverse immune microenvironments of sarcomas-including aspects not assessed in this study, such as macrophage and antigen presenting cell infiltration, plasma cells, and immune signalling networks-should be able to inform pragmatic designs of much-needed new clinical trials for the treatment of sarcomas.

Acknowledgements This study and international consortium are funded by the Liddy Shriver Sarcoma Initiative "ImmunoSarc" International Collaborative Grant. Dr. EGD would like to thank the Biorepository and Pathology Core at the Icahn School of Medicine at Mount Sinai, New York, NY, USA, for specimen retrieval, histology services, and tissue microarray construction. We thank Sonja Steigen-Ericsson for providing access to TMA06-001.

\section{Compliance with ethical standards}

Conflict of interest The authors declare that they have no conflict of interest.

Publisher's note: Springer Nature remains neutral with regard to jurisdictional claims in published maps and institutional affiliations.

\section{References}

1. Fletcher CDM, Bridge JA, Hogendoorn P, Mertens F. WHO classification of tumours of soft tissue and bone. In: WHO classification of tumours. 4th ed. Lyon, France: World Health Organization; 2013. volume 5. p. 10-1.

2. Young RJ, Natukunda A, Litière S, Woll PJ, Wardelmann E, van der Graaf WTA. First-line anthracycline-based chemotherapy for angiosarcoma and other soft tissue sarcoma subtypes: pooled analysis of eleven European Organisation for Research and Treatment of Cancer Soft Tissue and Bone Sarcoma Group trials. Eur J Cancer. 2014;50:3178-86.

3. Isakoff MS, Bielack SS, Meltzer P, Gorlick R. Osteosarcoma: current treatment and a collaborative pathway to success. J Clin Oncol. 2015;33:3029-35.

4. Treasure T, Fiorentino F, Scarci M, Møller H, Utley M. Pulmonary metastasectomy for sarcoma: a systematic review of reported outcomes in the context of Thames Cancer Registry data. BMJ Open. 2012;2:e001736.

5. In GK, Hu JS, Tseng WW. Treatment of advanced, metastatic soft tissue sarcoma: latest evidence and clinical considerations. Ther Adv Med Oncol. 2017;9:533-50. 
6. Le Cesne A, Blay JY, Judson I, Van Oosterom A, Verweij J, Radford J, et al. Phase II study of ET-743 in advanced soft tissue sarcomas: a European Organisation for the Research and Treatment of Cancer (EORTC) Soft Tissue and Bone Sarcoma Group trial. J Clin Oncol. 2005;23:576-84.

7. Nielsen OS, Judson I, Van Hoesel Q, Le Cesne A, Keizer HJ, Blay $\mathrm{JY}$, et al. Effect of high-dose ifosfamide in advanced soft tissue sarcomas. A multicentre phase II study of the EORTC Soft Tissue and Bone Sarcoma Group. Eur J Cancer. 2000;36:61-67.

8. Maki RG, Wathen JK, Patel SR, Priebat DA, Okuno SH, Samuels B, et al. Randomized phase II study of gemcitabine and docetaxel compared with gemcitabine alone in patients with metastatic soft tissue sarcomas: results of sarcoma alliance for research through collaboration study 002 [corrected]. J Clin Oncol. 2007;25:2755-63.

9. Leahy M, Garcia Del Muro X, Reichardt P, Judson I, Staddon A, Verweij J, et al. Chemotherapy treatment patterns and clinical outcomes in patients with metastatic soft tissue sarcoma. The sarcoma treatment and burden of illness in North America and Europe (SABINE) study. Ann Oncol. 2012;23:2763-70.

10. Ryan CW, Merimsky O, Agulnik M, Blay JY, Schuetze SM, Van Tine BA, et al. PICASSO III: a phase III, placebocontrolled study of doxorubicin with or without palifosfamide in patients with metastatic soft tissue sarcoma. J Clin Oncol. 2016;34:3898-905.

11. Savina M, Le Cesne A, Blay JY, Ray-Coquard I, Mir O, Toulmonde M, et al. Patterns of care and outcomes of patients with METAstatic soft tissue SARComa in a real-life setting: the METASARC observational study. BMC Med. 2017;15:78.

12. Dudley ME, Wunderlich JR, Robbins PF, Yang JC, Hwu P, Schwartzentruber DJ, et al. Cancer regression and autoimmunity in patients after clonal repopulation with antitumor lymphocytes. Science. 2002;298:850-4.

13. Dudley ME, Yang JC, Sherry R, Hughes MS, Royal R, Kammula $\mathrm{U}$, et al. Adoptive cell therapy for patients with metastatic melanoma: evaluation of intensive myeloablative chemoradiation preparative regimens. J Clin Oncol. 2008;26:5233-9.

14. Porter DL, Levine BL, Kalos M, Bagg A, June CH. Chimeric antigen receptor-modified $\mathrm{T}$ cells in chronic lymphoid leukemia. N Engl J Med. 2011;365:725-33.

15. Robbins PF, Morgan RA, Feldman SA, Yang JC, Sherry RM, Dudley ME, et al. Tumor regression in patients with metastatic synovial cell sarcoma and melanoma using genetically engineered lymphocytes reactive with NY-ESO-1. J Clin Oncol. 2011;29:917-24.

16. Robbins PF, Kassim SH, Tran TLN, Crystal JS, Morgan RA, Feldman SA, et al. A pilot trial using lymphocytes genetically engineered with an NY-ESO-1-reactive T-cell receptor: long-term follow-up and correlates with response. Clin Cancer Res. 2015;21:1019-27.

17. Pollack SM, Lu H, Gnjatic S, Somaiah N, O'Malley RB, Jones RL, et al. First-in-human treatment with a dendritic cell-targeting lentiviral vector-expressing NY-ESO-1, LV305, induces deep, durable response in refractory metastatic synovial sarcoma patient. J Immunother. 2017;40:302-6.

18. Somaiah N, Chawla SP, Block MS, Morris JC, Do KT, Kim JW, et al. Immune response, safety, and survival impact from CMB305 in NY-ESO-1+ recurrent soft tissue sarcomas (STS). J Clin Oncol. 2017;35:11006

19. Hodi FS, O'Day SJ, McDermott DF, Weber RW, Sosman JA, Haanen JB, et al. Improved survival with ipilimumab in patients with metastatic melanoma. N Engl J Med. 2010;363:711-23.

20. Wolchok JD, Neyns B, Linette G, Negrier S, Lutzky J, Thomas L, et al. Ipilimumab monotherapy in patients with pretreated advanced melanoma: a randomised, double-blind, multicentre, phase 2, dose-ranging study. Lancet Oncol. 2010;11:155-64.
21. Robert C, Thomas L, Bondarenko I, O’Day S, Weber J, Garbe C, et al. Ipilimumab plus dacarbazine for previously untreated metastatic melanoma. N Engl J Med. 2011;364:2517-26.

22. Topalian SL, Hodi FS, Brahmer JR, Gettinger SN, Smith DC, McDermott DF, et al. Safety, activity, and immune correlates of anti-PD-1 antibody in cancer. N Engl J Med. 2012;366:2443-54.

23. Ott PA, Elez E, Hiret S, Kim D-W, Morosky A, Saraf S, et al. Pembrolizumab in patients with extensive-stage small-cell lung cancer: results from the phase Ib KEYNOTE-028 study. J Clin Oncol. 2017;35:3823-9.

24. Herbst RS, Baas P, Kim D-W, Felip E, Pérez-Gracia JL, Han J-Y, et al. Pembrolizumab versus docetaxel for previously treated, PDL1-positive, advanced non-small-cell lung cancer (KEYNOTE010): a randomised controlled trial. Lancet. 2016;387:1540-50.

25. Plimack ER, Bellmunt J, Gupta S, Berger R, Chow LQM, Juco $\mathrm{J}$, et al. Safety and activity of pembrolizumab in patients with locally advanced or metastatic urothelial cancer (KEYNOTE012): a non-randomised, open-label, phase 1b study. Lancet Oncol. 2017;18:212-20.

26. Tawbi HA, Burgess M, Bolejack V, Van Tine BA, Schuetze SM, $\mathrm{Hu}$ J, et al. Pembrolizumab in advanced soft-tissue sarcoma and bone sarcoma (SARC028): a multicentre, two-cohort, single-arm, open-label, phase 2 trial. Lancet Oncol. 2017;18:1493-501.

27. Ben-Ami E, Barysauskas CM, Solomon S, Tahlil K, Malley R, Hohos M, et al. Immunotherapy with single agent nivolumab for advanced leiomyosarcoma of the uterus: results of a phase 2 study. Cancer. 2017;123:3285-90.

28. Maki RG, Jungbluth AA, Gnjatic S, Schwartz GK, D’Adamo DR, Keohan ML, et al. A pilot study of anti-CTLA4 antibody ipilimumab in patients with synovial sarcoma. Sarcoma. 2013;2013:168145-8.

29. Paoluzzi L, Cacavio A, Ghesani M, Karambelkar A, Rapkiewicz A, Weber J, et al. Response to anti-PD1 therapy with nivolumab in metastatic sarcomas. Clin Sarcoma Res. 2016;6:24.

30. Toulmonde M, Penel N, Adam J, Chevreau C, Blay JY, Le Cesne A, et al. Use of PD-1 targeting, macrophage infiltration, and IDO pathway activation in sarcomas: a phase 2 clinical trial. JAMA Oncol. 2018;4:93-97.

31. D'Angelo SP, Mahoney MR, Van Tine BA, Atkins J, Milhem MM, Jahagirdar BN, et al. Nivolumab with or without ipilimumab treatment for metastatic sarcoma (Alliance A091401): two openlabel, non-comparative, randomised, phase 2 trials. Lancet Oncol. 2018;19:416-26.

32. D'Angelo SP, Shoushtari AN, Agaram NP, Kuk D, Qin L-X, Carvajal RD, et al. Prevalence of tumor-infiltrating lymphocytes and PD-L1 expression in the soft tissue sarcoma microenvironment. Hum Pathol. 2015;46:357-65.

33. Kim C, Kim EK, Jung H, Chon HJ, Han JW, Shin K-H, et al. Prognostic implications of PD-L1 expression in patients with soft tissue sarcoma. BMC Cancer. 2016;16:434.

34. Kostine M, Cleven AH, de Miranda NFCC, Italiano A, Cleton Jansen AM, JVMG Bovée. Analysis of PD-L1, T-cell infiltrate and HLA expression in chondrosarcoma indicates potential for response to immunotherapy specifically in the dedifferentiated subtype. Mod Pathol. 2016;29:1028-37.

35. Bertucci F, Finetti P, Perrot D, Leroux A, Collin F, Le Cesne A, et al. PDL1 expression is a poor-prognosis factor in soft-tissue sarcomas. Oncoimmunology. 2017;6:e1278100.

36. Jenkins RW, Barbie DA, Flaherty KT. Mechanisms of resistance to immune checkpoint inhibitors. Br J Cancer. 2018;118:9-16.

37. Datar I, Mani N, Henick BS, Wurtz A, Kaftan E, Herbst RS, et al. Measurement of PD-1, TIM-3 and LAG-3 protein in non-small cell lung carcinomas (NSCLCs) with acquired resistance to PD-1 axis blockers. J Clin Oncol. 2017;35:e14611.

38. Burugu S, Dancsok AR, Nielsen TO. Emerging targets in cancer immunotherapy. Semin Cancer Biol. 2017;52:39-52. 
39. Ngiow SF, Scheidt von B, Akiba H, Yagita H, Teng MWL, Smyth MJ. Anti-TIM3 antibody promotes T cell IFN- $\gamma$-mediated antitumor immunity and suppresses established tumors. Clin Cancer Res. 2011;71:3540-51.

40. Nielsen TO, Hsu FD, O'Connell JX, Gilks CB, Sorensen PHB, Linn S, et al. Tissue microarray validation of epidermal growth factor receptor and SALL2 in synovial sarcoma with comparison to tumors of similar histology. Am J Pathol. 2003;163:1449-56.

41. Ng TL, Gown AM, Barry TS, Cheang M. Nuclear beta-catenin in mesenchymal tumors. Mod Pathol. 2005;18:68-74.

42. Steigen SE, Straume B, Turbin D, Chan AKW, Leung S, Nielsen $\mathrm{TO}$, et al. Clinicopathologic factors and nuclear morphometry as independent prognosticators in KIT-positive gastrointestinal stromal tumors. J Histochem Cytochem. 2008;56:139-45.

43. Cheng H, Dodge J, Mehl E, Liu S, Poulin N, van de Rijn M, et al. Validation of immature adipogenic status and identification of prognostic biomarkers in myxoid liposarcoma using tissue microarrays. Hum Pathol. 2009;40:1244-51.

44. Pacheco M, Nielsen TO. Histone deacetylase 1 and 2 in mesenchymal tumors. Mod Pathol. 2011;25:222-30.

45. Endo M, Su L, Nielsen TO. Activating transcription factor 2 in mesenchymal tumors. Hum Pathol. 2014;45:276-84.

46. Endo M, de Graaff MA, Ingram DR, Lim S, Lev DC, Briaire-de Bruijn IH, et al. NY-ESO-1 (CTAG1B) expression in mesenchymal tumors. Mod Pathol. 2015;28:587-95.

47. Banito A, Li X, Laporte AN, Roe J-S, Sanchez-Vega F, Huang C$\mathrm{H}$, et al. The SS18-SSX oncoprotein hijacks KDM2B-PRC1.1 to drive synovial sarcoma. Cancer Cell. 2018;33:527-8.

48. Terry J, Saito T, Subramanian S, Ruttan C, Antonescu CR, Goldblum JR, et al. TLE1 as a diagnostic immunohistochemical marker for synovial sarcoma emerging from gene expression profiling studies. Am J Surg Pathol. 2007;31:240-6.

49. Demicco EG, Harms PW, Patel RM, Smith SC, Ingram D, Torres $\mathrm{K}$, et al. Extensive survey of STAT6 expression in a large series of mesenchymal tumors. Am J Clin Pathol. 2015;143:672-82.

50. Garon EB, Rizvi NA, Hui R, Leighl N, Balmanoukian AS, Eder JP, et al. Pembrolizumab for the treatment of non-small-cell lung cancer. N Engl J Med. 2015;372:2018-28.

51. Pirici D, Mogoanta L, Kumar-Singh S, Pirici I, Margaritescu C, Simionescu C, et al. Antibody elution method for multiple immunohistochemistry on primary antibodies raised in the same species and of the same subtype. J Histochem Cytochem. 2009;57:567-75.

52. Constantinidou A, Miah A, Pollack S, Jones RL. New drugs and clinical trial design in advanced sarcoma: have we made any progress? Future Oncol. 2013;9:1409-11.

53. Dancsok AR, Asleh-Aburaya K, Nielsen TO. Advances in sarcoma diagnostics and treatment. Oncotarget. 2017;8:7068-93.

54. Pollack SM, He Q, Yearley JH, Emerson R, Vignali M, Zhang Y, et al. T-cell infiltration and clonality correlate with programmed cell death protein 1 and programmed death-ligand 1 expression in patients with soft tissue sarcomas. Cancer. 2017;123:3291-304.

55. Han Q, Shi H, Liu F. CD163(+) M2-type tumor-associated macrophage support the suppression of tumor-infiltrating $\mathrm{T}$ cells in osteosarcoma. Int Immunopharmacol. 2016;34:101-6.

56. Laginestra MA, Tripodo C, Agostinelli C, Motta G, Hartmann S, Döring $\mathrm{C}$, et al. Distinctive histogenesis and immunological microenvironment based on transcriptional profiles of follicular dendritic cell sarcomas. Mol Cancer Res. 2017;15:541-52.

57. Nowicki TS, Akiyama R, Huang RR, Shintaku IP, Wang X, Tumeh PC, et al. Infiltration of CD8 T cells and expression of PD-1 and PD-L1 in synovial sarcoma. Cancer Immunol Res. 2017;5:118-26.

58. Paydas S, Bagir EK, Deveci MA, Gonlusen G. Clinical and prognostic significance of PD-1 and PD-L1 expression in sarcomas. Med Oncol. 2016;33:93.
59. Torabi A, Amaya CN, Wians FH Jr., Bryan BA. PD-1 and PD-L1 expression in bone and soft tissue sarcomas. Mod Pathol. 2017:49:506-13.

60. Fritzsching B, Fellenberg J, Moskovszky L, Sápi Z, Krenacs T, Machado I, et al. CD8(+)/FOXP3(+)-ratio in osteosarcoma microenvironment separates survivors from non-survivors: a multicenter validated retrospective study. Oncoimmunology. 2015;4:e990800.

61. Machado I, López-Guerrero JA, Scotlandi K, Picci P, LlombartBosch A. Immunohistochemical analysis and prognostic significance of PD-L1, PD-1, and CD8+ tumor-infiltrating lymphocytes in Ewing's sarcoma family of tumors (ESFT). Virchows Arch. 2018;472:815-24.

62. Boxberg M, Steiger K, Lenze U, Rechl H, Eisenhart-Rothe von R, Wörtler K, et al. PD-L1 and PD-1 and characterization of tumorinfiltrating lymphocytes in high grade sarcomas of soft tissue prognostic implications and rationale for immunotherapy. Oncoimmunology. 2018;7:e1389366.

63. Shurell E, Singh AS, Crompton JG, Jensen S, Li Y, Dry S, et al. Characterizing the immune microenvironment of malignant peripheral nerve sheath tumor by PD-L1 expression and presence of CD8+ tumor infiltrating lymphocytes. Oncotarget. 2016;7:64300-8.

64. van Erp AEM, Versleijen-Jonkers YMH, Hillebrandt-Roeffen MHS, van Houdt L, Gorris MAJ, van Dam LS, et al. Expression and clinical association of programmed cell death-1, programmed death-ligand-1 and CD8+ lymphocytes in primary sarcomas is subtype dependent. Oncotarget. 2017;8:71371-84.

65. Spurny C, Kailayangiri S, Jamitzky S, Altvater B, Wardelmann E, Dirksen U, et al. Programmed cell death ligand 1 (PD-L1) expression is not a predominant feature in Ewing sarcomas. Pediatr Blood Cancer. 2018;65:e26719.

66. Simard FA, Richert I, Vandermoeten A, Decouvelaere A-V, Michot J-P, Caux C, et al. Description of the immune microenvironment of chondrosarcoma and contribution to progression. Oncoimmunology. 2017;6:e1265716.

67. Rusakiewicz S, Semeraro M, Sarabi M, Desbois M, Locher C, Mendez R, et al. Immune infiltrates are prognostic factors in localized gastrointestinal stromal tumors. Clin Cancer Res. 2013;73:3499-510.

68. D'Angelo SP, Shoushtari AN, Keohan ML, Dickson MA, Gounder MM, Chi P, et al. Combined KIT and CTLA-4 blockade in patients with refractory GIST and other advanced sarcomas: a phase Ib study of dasatinib plus ipilimumab. Clin Cancer Res. 2017;23:2972-80.

69. Thorsson V, Gibbs DL, Brown SD, Wolf D, Bortone DS, Ou Yang T-H, et al. The immune landscape of cancer. Immunity. 2018;48:812-4.

70. Cancer Genome Atlas Research Network. Comprehensive and integrated genomic characterization of adult soft tissue sarcomas. Cell. 2017;171:950-65. Electronic address: elizabeth.demicco@sinaihealthsystem.ca, Cancer Genome Atlas Research Network.

71. Merchant MS, Wright M, Baird K, Wexler LH, RodriguezGalindo C, Bernstein D, et al. Phase I clinical trial of ipilimumab in pediatric patients with advanced solid tumors. Clin Cancer Res. 2016;22:1364-70.

72. Ghisoli M, Barve M, Mennel R, Lenarsky C, Horvath S, Wallraven $\mathrm{G}$, et al. Three-year follow up of GMCSF/bi-shRNA furin DNAtransfected autologous tumor immunotherapy (vigil) in metastatic advanced Ewing's sarcoma. Mol Ther. 2016;24:1478-83.

73. Miwa S, Nishida H, Tanzawa Y, Takeuchi A, Hayashi K, Yamamoto $\mathrm{N}$, et al. Phase $1 / 2$ study of immunotherapy with dendritic cells pulsed with autologous tumor lysate in patients with refractory bone and soft tissue sarcoma. Cancer. 2017;123:1576-84

74. Pollack SM. The potential of the CMB305 vaccine regimen to target NY-ESO-1 and improve outcomes for synovial sarcoma and 
myxoid/round cell liposarcoma patients. Expert Rev Vaccines. 2018;17:107-14.

75. Research AAFC. High TMB predicts immunotherapy benefit. Cancer Discov. 2018;8:668.

76. Hellmann MD, Ciuleanu T-E, Pluzanski A, Lee JS, Otterson GA, Audigier-Valette $\mathrm{C}$, et al. Nivolumab plus ipilimumab in lung cancer with a high tumor mutational burden. $\mathrm{N}$ Engl J Med. 2018;378:2093-104.

77. Hellmann MD, Callahan MK, Awad MM, Calvo E, Ascierto PA, Atmaca A, et al. Tumor mutational burden and efficacy of nivolumab monotherapy and in combination with ipilimumab in smallcell lung cancer. Cancer Cell. 2018;33:853-4.

78. Liao Y, Chen L, Feng Y, Shen J, Gao Y, Cote G, et al. Targeting programmed cell death ligand 1 by CRISPR/Cas9 in osteosarcoma cells. Oncotarget. 2017;8:30276-87.

79. Kim JR, Moon YJ, Kwon KS, Bae JS, Wagle S, Kim KM. et al. Tumor infiltrating PD1-positive lymphocytes and the expression of PD-L1 predict poor prognosis of soft tissue sarcomas. PLoS ONE. 2013;8:e82870. Lee J-S, editor.

80. Costa Arantes DA, Gonçalves AS, Jham BC, Duarte ECB, de Paula ÉC, de Paula HM, et al. Evaluation of HLA-G, HLA-E, and PD-L1 proteins in oral osteosarcomas. Oral Surg Oral Med Oral Pathol Oral Radio. 2017;123:e188-196.

81. Chowdhury F, Dunn S, Mitchell S, Mellows T, Ashton-Key M, Gray JCPD-L1. and CD8+PD1+ lymphocytes exist as targets in the pediatric tumor microenvironment for immunomodulatory therapy. Oncoimmunology. 2015;4:e1029701.

82. Que Y, Xiao W, Guan Y-X, Liang Y, Yan S-M, Chen H-Y, et al. PD-L1 expression is associated with FOXP3 + regulatory T-cell infiltration of soft tissue sarcoma and poor patient prognosis. J Cancer. 2017;8:2018-25.

83. Budczies J, Mechtersheimer G, Denkert C, Klauschen F, Mughal SS, Chudasama P, et al. PD-L1 (CD274) copy number gain, expression, and immune cell infiltration as candidate predictors for response to immune checkpoint inhibitors in soft-tissue sarcoma. Oncoimmunology. 2017;6:e1279777.

84. Sundara YT, Kostine M, Cleven AHG, JVMG Bovée, Schilham MW, Cleton Jansen AM. Increased PD-L1 and T-cell infiltration in the presence of HLA class I expression in metastatic high-grade osteosarcoma: a rationale for T-cell-based immunotherapy. Cancer Immunol Immunother. 2017;66:119-28.

85. Shen JK, Cote GM, Choy E, Yang P, Harmon D, Schwab J, et al. Programmed cell death ligand 1 expression in osteosarcoma. Cancer Immunol Res. 2014;2:690-8.
86. Koirala P, Roth ME, Gill J, Piperdi S, Chinai JM, Geller DS, et al. Immune infiltration and PD-L1 expression in the tumor microenvironment are prognostic in osteosarcoma. Sci Rep. 2016;6:30093.

87. Honda Y, Otsuka A, Ono S, Yamamoto Y, Seidel JA, Morita S, et al. Infiltration of PD-1-positive cells in combination with tumor site PD-L1 expression is a positive prognostic factor in cutaneous angiosarcoma. Oncoimmunology. 2017;6:e1253657.

88. Calles A, Liao X, Sholl LM, Rodig SJ, Freeman GJ, Butaney M, et al. Expression of PD-1 and its ligands, PD-L1 and PD-L2, in smokers and never smokers with KRAS-mutant lung cancer. J Thorac Oncol. 2015;10:1726-35.

89. Krawczyk P, Jarosz B, Kucharczyk T, Grenda A, Reszka K, Pankowski $\mathrm{J}$, et al. Immunohistochemical assays incorporating SP142 and 22C3 monoclonal antibodies for detection of PD-L1 expression in NSCLC patients with known status of EGFR and ALK genes. Oncotarget. 2017;8:64283-93.

90. Xu H, Lin G, Huang C, Zhu W, Miao Q, Fan X, et al. Assessment of concordance between 22C3 and SP142 immunohistochemistry assays regarding PD-L1 expression in non-small cell lung cancer. Sci Rep. 2017;7:16956.

91. Woo S-R, Turnis ME, Goldberg MV, Bankoti J, Selby M, Nirschl $\mathrm{CJ}$, et al. Immune inhibitory molecules LAG-3 and PD-1 synergistically regulate $\mathrm{T}$-cell function to promote tumoral immune escape. Clin Canc Res. 2012;72:917-27.

92. Huang R-Y, Francois A, McGray AR, Miliotto A, Odunsi K. Compensatory upregulation of PD-1, LAG-3, and CTLA-4 limits the efficacy of single-agent checkpoint blockade in metastatic ovarian cancer. Oncoimmunology. 2017;6:e1249561.

93. Shayan G, Srivastava R, Li J, Schmitt N, Kane LP, Ferris RL. Adaptive resistance to anti-PD1 therapy by Tim-3 upregulation is mediated by the PI3K-Akt pathway in head and neck cancer. Oncoimmunology. 2017;6:e1261779.

94. Koyama S, Akbay EA, Li YY, Herter-Sprie GS, Buczkowski KA, Richards WG, et al. Adaptive resistance to therapeutic PD-1 blockade is associated with upregulation of alternative immune checkpoints. Nat Commun. 2016;7:10501.

95. Gameiro SR, Jammeh ML, Wattenberg MM, Tsang KY, Ferrone S, Hodge JW. Radiation-induced immunogenic modulation of tumor enhances antigen processing and calreticulin exposure, resulting in enhanced T-cell killing. Oncotarget. 2014;5:403-16.

96. Golden EB, Apetoh L. Radiotherapy and immunogenic cell death. Semin Radiat Oncol. 2015;25:11-17.

97. Ng J, Dai T. Radiation therapy and the abscopal effect: a concept comes of age. Ann Transl Med. 2016;4:118. 